\title{
Evidence for functional and non-functional classes of peptides translated from long non-coding RNAs
}

Jorge Ruiz-Orera ${ }^{1, *}$, Pol Verdaguer-Grau ${ }^{2}$, José Luis Villanueva-Cañas ${ }^{1}$, Xavier Messeguer ${ }^{2}$, M.Mar Albà ${ }^{1,3, *}$

${ }^{1}$ Evolutionary Genomics Group, Research Programme on Biomedical Informatics, Hospital del Mar Research Institute, Universitat Pompeu Fabra, Barcelona, Spain; ${ }^{2}$ Computer Sciences Department, Universitat Politècnica de Catalunya, Barcelona, Spain; ${ }^{3}$ Catalan Institution for Research and Advanced Studies, Barcelona, Spain.

*To whom correspondence should be addressed.

Running title: pervasive translation

Keywords: ribosome profiling, translation, de novo gene, long non-coding RNA, peptide, polymorphism, natural selection 


\section{Abstract}

2

3 There is accumulating evidence that some genes have originated de novo from previously non-

4 coding genomic sequences. However, the processes underlying de novo gene birth are still

5 enigmatic. In particular, the appearance of a new functional protein seems highly improbable

6 unless there is already a pool of neutrally evolving peptides that can at some point acquire new

7 functions. Here we show for the first time that such peptides do not only exist but that they are

8 prevalent among the translation products of mouse genes that lack homologues in rat and

9 human. The data suggests that the translation of these peptides is due to the chance

10 occurrence of open reading frames with a favorable codon composition. Our approach

11 combines ribosome profiling experiments, proteomics data and non-synonymous and

12 synonymous nucleotide polymorphism analysis. We propose that effectively neutral processes

13 involving the expression of thousands of transcripts all the way down to proteins provide a basis

14 for de novo gene evolution. 
15 The mammalian genome is pervasively transcribed, this includes functional genes but also

16 thousands of transcripts that are not conserved across species and which show weak or no

17 signatures of natural selection ${ }^{1-3}$. Many of the latter transcripts are annotated as long non-

18 coding RNAs (IncRNAs) because they lack conserved long open reading frames (ORFs).

19 Recent studies based on the sequencing of ribosome-protected RNA fragments (ribosome

20 profiling) have reported that a surprisingly large fraction of these transcripts is likely to translate

21 small peptides ${ }^{4-9}$, although the significance of this finding has remained elusive.

22

23 Each ribosome profiling experiment generates millions of ribosome footprints that are

24 subsequently mapped to the genome or the transcriptome to identify open reading frames

25 (ORFs) that are being translated ${ }^{10}$. The codon-by-codon movement of the ribosome along the

26 coding sequence results in a characteristic pattern of three nucleotide periodicity of the mapped

27 reads, which makes ribosome profiling a very useful method to detect novel events of

28 translation ${ }^{4,11,12}$. Given enough sequence coverage the technique can uncover low-abundant

29 small peptides that would be otherwise difficult to detect by standard proteomics

30 approaches ${ }^{13,14}$.

32 To assess the functional relevance of novel events of translation one can use the ratio between

33 the number of non-synonymous and synonymous substitutions in the putative coding

34 sequences ${ }^{4,5}$. However, this method requires an alignment of at least two homologous

35 sequences. A more general approach that can be used in the absence of homology is the ratio

36 between the number of non-synonymous and synonymous single nucleotide polymorphisms,

37 compared to the one expected under neutrality. Under no selection, non-synonymous and

38 synonymous polymorphisms accumulate at the same rate, whereas under purifying selection

39 there is a deficit of non-synonymous polymorphisms because some amino acid changes disrupt

40 the protein's function ${ }^{15}$. Single nucleotide polymorphism analysis can be performed on a gene-

41 by-gene basis or in pools of sequences that share certain features ${ }^{2,16}$.

42

43 We previously observed that, as a whole, putatively translated IncRNAs and young protein-

44 coding genes share a number of similarities, such as small ORF size and weak selective 
45 constraints, compared with more widely conserved genes ${ }^{8}$. This pointed to a link between the

46 translation of IncRNAs and the evolution of new proteins, but it did not solve the key question of

47 whether translation of new ORFs could occur in the absence of selection at the protein level.

48 This is a fundamental issue because for a new protein to acquire a function it first needs to be

49 produced in the cell at significant amounts. Here by employing a combination of ribosome

50 profiling data, sequence analysis and single nucleotide polymorphism information we obtain

51 strong evidence that the majority of mouse proteins that are not conserved in rat or human

52 selection evolve in a neutral manner. This study renders visible for the first time a layer of

53 protein expression that is not dependent on selective processes, filling a gap in our

54 understanding of the processes underlying de novo gene birth.

\section{Results}

58 First we set to identify translated open reading frames (ORFs) in mouse protein-coding genes

59 (codRNAs) and long non-coding RNAs (IncRNAs) using ribosome-profiling RNA-sequencing

60 (Ribo-Seq) data from eight different tissues and cell lines (Supplementary Table 1 and

61 references therein). In contrast to RNA sequencing (RNA-Seq) reads, which are expected to

62 cover the complete transcript, Ribo-Seq reads correspond to regions bound by ribosomes. We

63 mapped the RNA-Seq and Ribo-Seq reads to the mouse Ensembl gene annotations and, for the

64 sake of completeness, also to a set of previously obtained novel mouse transcripts that did not

65 correspond to annotated genes ${ }^{3}$.

66 We used the RibORF program ${ }^{4}$ to identify bona fide translated sequences among ORFs

67 covered by at least 10 Ribo-Seq reads in transcripts expressed in one or more tissues (Fig. 1a

68 and Supplementary Table 1). This program calculates a score for each ORF depending on the

69 3-nucleotide periodicity and uniformity of the mapped reads. Using a highly stringent RibORF

70 score cut-off of $0.7^{4}$ we found that about $90 \%$ of the coding genes $(15,020)$, and $20 \%$ of the

71 annotated IncRNAs (539), were predicted to be translated in at least one sample. Additionally,

72 we identified 286 genes that did not map to the gene annotations but contained translated

73 ORFs (Fig. 1b). A widely used criterion to annotate a transcript as protein-coding is the 
74 presence of an ORF encoding a protein of at least 100 amino acids ${ }^{17}$. Not surprisingly, the vast

75 majority of ORFs translated from annotated and novel IncRNAs encoded proteins smaller than

76100 amino acids (smORFs).

77 We hypothesized that some of the translated ORFs may evolve in a neutral manner and

78 constitute a reservoir for the evolution of new protein-coding genes. To test this hypothesis, we

79 first identified translated ORFs that were mouse-specific and then tested them for signatures of

80 selection. We performed exhaustive sequence similarity searches of the ORFs against high

81 coverage transcriptomes from human and rat as well as against the annotated proteomes of

82101 different eukaryotic species (Fig. 2a, Supplementary Table 2 for a list of species, see

83 Methods for more details). For these searches we discarded any proteins shorter than 24 amino

84 acids, as the detection of homologues may be compromised in such cases due to lack of

85 sufficient sequence information. We identified 1,980 different translated ORFs that showed no

86 homology to expressed sequences in other species (class non-conserved or NC). In general,

87 these ORFs had lower codon usage bias than conserved ORFs, as measured by a previously

88 described hexamer-based coding score metric ${ }^{8}$ (Fig. 2b).

90 To measure the strength of selection in conserved and non-conserved translated ORFs we

91 employed a large collection of mouse single nucleotide polymorphisms (SNPs) for the house

92 mouse subspecies Mus musculus castaneus ${ }^{18}$. We could map a total of 324,729 SNPs to the

93 set of translated ORFs. We grouped the ORFs into three different classes on the basis of

94 conservation and coding score (Fig. 2b), and calculated the ratio between the number of

95 observed non-synonymous and synonymous SNPs (PN/PS(obs)) in each class. We then

96 normalized it by the same ratio expected under neutrality (PN/PS(exp)). The expected PN/PS

97 was estimated using a table of nucleotide mutation frequencies in Mus musculus castaneus and

98 the observed codon frequencies in each set of sequences of interest (Supplementary Tables 3

99 and 4). This allowed us not only to compare the strength of selection across different sets of

100 sequences, as done in a previous study of ORFs translated from IncRNAs ${ }^{8}$, but also to discard

101 selection if the normalized PN/PS was not significantly different from 1. Specifically, we used a

102 chi-square test that compared the number of observed and expected non-synonymous and

103 synonymous SNPs in each sequence set (Supplementary Table 5). As expected, the PN/PS of 
104 randomly selected ORFs from introns was approximately 1 . Instead, the PN/PS of conserved

105 ORFs was around 0.15 (Fig. 2c, chi-square test p-value $<10^{-5}$ ), consistent with protein

106 functionality. One example in this group was $\operatorname{Stannin}^{19,20}$, a highly conserved peptide that

107 regulates neuronal cell apoptosis (Fig. 3).

108

109 Non-conserved ORFs with high coding scores (NC-H coding score $\geq 0.1014$, Fig. 2b and c,

110 Supplementary Figure 1) had weak but significant signatures of selection ( $p$-value $<0.05$ ),

111 possibly because of the existence of some functional mouse-specific genes. In contrast, the

112 PN/PS ratio of the remaining non-conserved ORFs was not significantly different from 1 ,

113 consistent with neutral evolution. Very similar results were obtained for non-conserved genes

114 annotated as coding or IncRNA (Fig. 2c) and the two sets were merged into a single group of

115 neutrally evolving ORFs (neutral ORFs). This set comprised about two thirds of the non-

116 conserved ORFs ( 1,291 out of 1,980 ORFs analysed), and represented $\sim 6.8 \%$ of the total

117 number of mouse translated ORFs.

119 We used proteomics data from the PRIDE database ${ }^{21}$ to further validate the translation of this

120 latter group of proteins. Despite their small size (median 44 amino acids), a limiting factor for

121 their detection by standard proteomics-based tecniques ${ }^{22}$, we found proteomics evidence for 32

122 of the neutral ORFs (see Methods). This represents $2.5 \%$ of the proteins in this set (compared

123 to less than $0.2 \%$ false positive rate, see Methods). This fraction is similar to the one obtained

124 for conserved proteins subsampled to have a similar size distribution as the neutral ORFs

125 (2.9\%; in contrast, about $41 \%$ of all conserved ORFs have proteomics evidence). The test

126 based on the PN/PS ratio confirmed that this subset of 32 ORFs did not deviate significantly

127 from neutrality either (Supplementary Table 5).

129 The above analyses grouped the sequences into classes before computing the PN/PS ratio. In

130 general, ORF-by-ORF analysis was not possible because the ORFs were small and contained

131 too few SNPs. Nevertheless, 41 of the neutrally evolving ORFs contained 10 or more SNPs,

132 and we decided to compute a normalized PN/PS ratio for these individual cases. The median

133 PN/PS of these ORFs was around 1 and the distribution of PN/PS values was very different 
134 from that of conserved ORFs (Fig. 2d, Wilcoxon test, p-value $<10^{-5}$ ), consistent with the

135 previous results. Finally, we quantified the number of ORFs that contained SNPs that generated

136 premature stop codons, truncating more than half of the ORF, in the set of neutrally evolving

137 ORFs and in the set of conserved ORFs. In the first case we found 72 out of 1,282 ORFs that

138 contained this type of mutation (5.6\%) and in the second case 296 out of 16,892 ORFs $(1.75 \%)$.

139 Considering that neutral ORFs are in general much shorter than conserved ORFs (median

140 protein size 44 versus 412 amino acids), and thus less likely to accumulate ORF-truncating

141 mutations by chance alone, the data clearly indicates a strong excess of ORF-truncating SNPs

142 in neutral ORFs with respect to conserved ORFs. These analyses further support that the

143 selective pressures acting on both kinds of ORFs are very different.

145 We next inspected in more detail the ribosome profiling patterns of neutral ORFs with respect to

146 the rest of translated ORFs (hereafter called "functional"). Genes with a recent origin are usually

147 expressed at lower levels than older genes ${ }^{23,24,3}$, so it was not surprising to observe that

148 neutrally evolving ORFs were associated with a lower number of Ribo-Seq reads per base than

149 the rest of translated ORFs (median 0.193 versus 0.474 , respectively, Supplementary figure 2 ).

150 Consistent with translation, read periodicity in both neutral and functional ORFs was much

151 higher than the random expectation of 0.33 (median values 0.70 and 0.80 , respectively; see

152 examples in Figure 3). Importantly, the results were highly reproducible across tissues (Figure

153 4a for hippocampus and embryonic stem cells; Supplementary Figure 3 hippocampus and

154 brain), a result we would not expect in the case of spurious ribosome profiling signals. In

155 general, the RibORF score of the translated ORFs was positively related to the number of

156 mapped Ribo-Seq reads (Spearman correlation $\mathrm{R}=0.408$ ), and to the size of the ORFs

157 (Spearman correlation $\mathrm{R}=0.193$ ). When we controlled for these two parameters, neutral and

158 functional ORFs had equivalent distributions of RibORF score, periodicity and uniformity values

159 (Figure 4b).

160

161 Subsequently, we compared our results to those obtained with two negative controls. The first

162 control contained ORFs in alternative frames of annotated protein coding sequences with

163 experimental protein evidence ("off-frame"). The second one contained randomly occurring 
164 ORFs in small nuclear and nucleolar annotated RNA sequences ("sRNA"). The latter RNAs are

165 sometimes detected in ribosome profiling experiments due to the formation of ribonucleoprotein

166 particles that protect the RNA from degradation ${ }^{25}$. As before, we only considered ORFs with at

167 least 10 Ribo-Seq mapped reads. As expected, the vast majority of the ORFs in these controls

168 did not display significant 3-nucleotide read periodicity (Supplementary figure 2, see a specific

169 example in Figure 3). We found that only 234 out of 13,596 ORFs in "off-frame", and 10 out of

170304 ORFs in "sRNA", had a RibORF score $\geq 0.7$ (the threshold employed throughout our study).

171 This corresponds to an overall false discovery rate (FDR) of $1.75 \%$, much lower than the

172 fraction of neutrally evolving proteins detected in our main analysis $(6.8 \%)$.

173

174 Some transcripts contained relatively long ORFs but were not translated. One example of this

175 sort was the previously described de novo non-coding gene Poldi ${ }^{26}$ that lacked any evidence of

176 translation in the data we analysed. We next asked which factors may influence the translation

177 of some neutrally evolving ORFs but not of others. First, we inspected the translation initiation

178 sequence context but did not detect any significant differences between translated and non-

179 translated ORFs (Supplementary Figure 4). We then hypothesized that the ORF coding score

180 could affect the "translatability" of the transcript because codons that are abundant in coding

181 sequences are expected to be more efficiently translated than other codons. Consistent with

182 this hypothesis, we found that the translated neutrally evolving ORFs exhibited higher coding

183 scores than non-translated ORFs with otherwise similar characteristics (Fig. 5a, Translated

184 versus non-translated Wilcoxon test, $p$-value $<10^{-5}$ ). Importantly, we obtained a similar result

185 after controlling for gene expression level (Fig. 5b, Wilcoxon test, p-value $<10^{-5}$ ). This is

186 consistent with codon composition having an effect per se in ORF translation. When controlling

187 by coding score, expression level, but not ORF length, had an effect on the translatability of the

188 transcript (Fig. 5c).

190 The results suggest that the neutral ORFs that are translated are enriched in codons that are

191 frequently found in functional protein coding sequences. This is consistent with the observation

192 that abundant codons enhance translation elongation ${ }^{27}$, whereas rare codons might affect the

193 stability of the $\mathrm{mRNA}^{28}$. It has been previously hypothesized that the distinction between 
194 translated and non-translated IncRNAs may be related to the relative amount of the IncRNA in

195 the nucleus and the cytoplasm ${ }^{4}$. However, we found evidence that some IncRNAs with nuclear

196 functions, such as Malat1 and Neat1, were translated, suggesting that the cytosolic fraction of a

197 transcript can be translated independently of its role or preferred location.

\section{DISCUSSION}

202 The molecular mechanisms underlying de novo gene evolution are still poorly understood $\mathrm{d}^{29,30,31}$.

203 The sudden appearance of a new protein-coding gene from a genomic segment seems a priori 204 highly improbable, but the process becomes much more likely if the genome is already being 205 pervasively transcribed and translated outside functional protein-coding genes. An excess of 206 transcription was already noted in the first large-scale cDNA sequencing efforts performed in 207 human and mouse ${ }^{32}$, and more recent studies have found a high rate of transcriptional turnover 208 when comparing closely related species ${ }^{33}$. Here we have shown that many of these transcripts 209 are translated even if they only contain small ORFs, with the data currently available we have 210 been able to identify 1,291 peptides in 1,132 genes that are likely to be of recent evolutionary 211 origin and that show no signs of selection. This number is likely to be a gross underestimate 212 because many transcripts are expressed at low levels, limiting their detection, and many cell 213 types and tissues have not yet been sampled. According to recent estimates, the cost of 214 transcription and translation in multicellular organisms is probably too small to overcome genetic $215 \mathrm{drift}^{34}$. Therefore, these activities may be effectively neutral. Our results indeed support that 216 there is no barrier for the production of peptides that do not confer an immediate selective 217 advantage.

219 The putative precursors of novel proteins identified here are of small size, which is consistent 220 with observations for functional de novo genes identified in previous studies ${ }^{23,35-37}$. We have 221 also shown that random ORFs with a more favorable, coding-like, hexamer composition are 222 more likely to be translated than other ORFs. Codon usage bias in functional sequences is 223 related to the abundance of different tRNAs and correlates with expression level ${ }^{38,39}$. Thus, it 
224 seems logical that the translated neutral ORFs are biased towards those codons that are

225 translated more efficiently.

226

227 The process of de novo gene origination involves the gain of a useful function by a previously

228 non-functional sequence. The rate at which this happens remains to be determined but it has

229 been observed that many random peptide sequences can function as secretion signals ${ }^{40}$, and

230 selection for ATP-binding activity in a library of randomly generated 80 amino acid polypeptides

231 successfully identified several candidates capable of binding to ATP ${ }^{41}$. Recent experiments

232 performed in E.coli also suggest that random sequences can often affect cellular growth ${ }^{42}$. The

233 pervasive translation of the transcriptome implies that de novo gene evolution has much more

234 material at its disposal than previously thought.

235

236

\section{METHODS}

\section{Transcript assembly}

241 We used strand-specific polyA+RNA sequencing data (RNA-Seq) data from different mouse

242 and human tissues to assembly the species transcriptomes (Gene Expression Omnibus mouse

243 GSE69241 ${ }^{3}$, GSE43721 43 , and GSE43520 44 ; human GSE69241 $1^{3}$ ). The mouse RNA samples

244 were extracted from strain Balb/C. RNA-Seq reads were filtered by length (> 25 nucleotides)

245 and by quality using Condetri $(\mathrm{v} \cdot 2.2)^{45}$ with the following settings: $-\mathrm{hq}=30-\mathrm{lq}=10$. We aligned

246 the reads to the corresponding reference species genome with Tophat (v. 2.0.8, $-\mathrm{N} 3$, -a 5 and

$247-\mathrm{m} 1^{46}$. Multiple mapping to several locations in the genome was allowed unless otherwise

248 stated. We assembled the transcriptome with Stringtie $^{47}$, merging the reads from all the

249 samples, with parameters -f 0.01 , and $-\mathrm{M} 0.2$. We used the species transcriptome as a guide

250 (Ensembl v.75), including all annotated isoforms, but permitting the assembly of annotated and

251 novel isoforms and genes (antisense, intergenic and intronic) as well. We excluded IncRNAs

252 that overlapped annotated pseudogenes or that showed significant sequence similarity to

253 known protein-coding sequences (BLASTP, e-value $<10^{-4}$ ). In the case of rat we employed a 
254 previously generated transcript assembly ${ }^{48}$.

255

\section{Ribosome profiling data}

258 We used ribosome profiling data (Ribo-Seq) from 8 different mouse tissues or cell lines (see 259 Supplementary Table 1), obtained from Gene Expression Omnibus under accession numbers

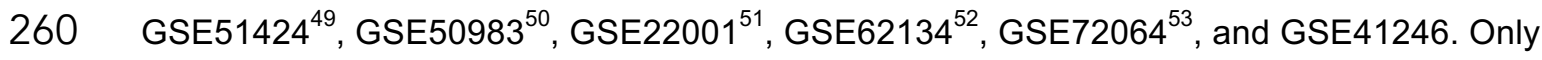
261 datasets corresponding to non-pathogenic conditions were considered. The reads from the 262 experimental replicates were merged before using RibORF to increase the resolution of the 263 read periodicity, as done in the original RibORF paper ${ }^{4}$. For all analyses we considered only 264 genes expressed at significant levels in at least one sample (RNA-Seq fragments per kilobase 265 per Million mapped reads $(F P K M)>0.2)$. The expression of the genes detected in these 266 samples is expected to be highly representative of the Mus musculus species as a whole. We 267 mapped several brain RNA-Seq datasets from Mus musculus castaneus ${ }^{33}$ to the mouse 268 assembled transcriptome using NextGenMap ${ }^{54}$. As expected, the vast majority of the genes 269 expressed in brain samples from C57BL/6 mice ${ }^{49}$ also showed evidence of expression in Mus 270 musculus castaneus brain RNA samples ${ }^{33}$ (Supplementary Table 6).

272 We discarded anomalous reads (length $<26$ or $>33 \mathrm{nt}$ ) and reads that mapped to annotated 273 rRNAs and tRNAs in mouse from the Ribo-Seq sequencing datasets. Next, reads were mapped 274 to the assembled mouse genome (mm10) with Bowtie (v. 0.12.7, parameters -k $1-\mathrm{m} 20-\mathrm{n} 1--$ 275 best --strata). Considering that the ORFs had to be extensively covered by reads to be 276 considered translated (high uniformity), we decided to include multiple mapped reads so as not 277 to compromise the detection of paralogous proteins (Supplementary Fig. 7). We used the 278 mapping of the Ribo-Seq reads to the complete set of annotated coding sequences in mouse to 279 compute the position of the P-site (second binding site for tRNA in the ribosome) for reads of 280 different size, as previously described ${ }^{10,12}$.

\section{Identification of translated ORFs}


284 We predicted all translated ORFs (ATG to STOP) with a minimum length of 9 amino acids in the

285 transcripts with RibORF (v.0.1) $)^{4}$. Only ORFs with a minimum of 10 mapped Ribo-Seq reads

286 were considered. The RibORF classifier is based on a support vector machine algorithm,

287 originally applied to human transcripts. The input parameters are the read periodicity and the

288 read uniformity. The first one is the fraction of reads that correspond to the correct frame and

289 the second one corresponds to the percentage of maximum entropy, a value of 1 indicates a

290 completely even distribution of reads. For each ORF the program computes a score that

291 depends on the values of these two parameters ${ }^{4}$. We used the same score cut-off as in the

292 original paper $(\geq 0.7$ ), which had a reported false positive rate of $0.67 \%$ and false negative rate

293 of $2.5 \%$.

294

295 We eliminated any redundancy in the translated ORFs by taking the longest ORF when several

296 overlapping translated ORFs were detected in the same gene. The identification of translated

297 ORFs was done separately for the different tissues (Supplementary Table 1), and the data was

298 subsequently integrated, taking the tissue with the highest RibORF score as representative.

299 Differences in the number of translated ORFs in different tissues were related to the depth of

300 sequencing and the number of reads that mapped to the top 5 most highly expressed proteins

301 (Supplementary Fig. 6 and 7, respectively). For genes with no evidence of translation we

302 selected the longest ORF across all transcripts for comparative purposes. Selecting the longest

303 ORF was justified by the fact that, in translated ORFs, the ORF with the highest number of

304 mapped Ribo-Seq reads was usually the longest ORF (75.7\% for codRNAs and $84 \%$ for

305 IncRNAs). We also generated a set of 4,013 randomly taken ORFs from introns, after discarding

306 ORFs that showed significant sequence similarity to known proteins from the same species

307 (BLASTP, e-value $<10^{-4}$ ).

308

309 We generated a negative control set by combining out-of-frame ORFs in mouse coding genes

310 with experimental protein evidence according to Uniprot ("off-frame") and randomly occurring

311 ORFs in mouse small nuclear and nucleolar RNAs ("sRNAs"). These ORFs were required to

312 have at least 10 Ribo-Seq mapped reads and were processed in the same manner as the main

313 set of ORFs under study. The total number of sequences in the negative control was 13,900 . 
314 We also generated a positive control set composed of 2,163 randomly taken annotated mouse

315 coding sequences with protein evidence in Uniprot. With these controls we estimated a false

316 positive rate of $1.75 \%$ and a false negative rate of $2.54 \%$ for the above mentioned RibORF

317 score cut-off.

\section{Sequence conservation}

321 We searched for mouse translated ORF homologues in the human and rat transcriptomes using

322 TBLASTN (limited to one strand, e-value $\left.<10^{-4}\right)^{55}$. We also performed sequence similarity

323 searches against the annotated proteomes of 67 mammalian-species and 34 non-mammalian

324 eukaryotes from a diverse range of groups compiled in a previous study ${ }^{48}$, using BLASTP (e-

325 value $<10^{-4}$ ). For these searches we only considered query proteins of size 24 amino acids or

326 longer, as shorter proteins may not contain sufficient information to perform homology searches.

327 Mouse ORFs that did not have any homology hits in other species were classified as non-

328 conserved, the rest as conserved. Translated non-conserved ORFs located upstream or

329 downstream of another longer ORF in a conserved transcript (uORFs and dORFs) were

330 excluded from this analysis.

332 We inspected the rat genomic syntenic regions of translated ORFs using LiftOver ${ }^{56}$. We

333 classified the ORFs in two groups depending on whether the ORF was truncated in rat or not

334 (the truncation had to affect more than half of the protein). For neutrally evolving ORFs the

335 number of cases in which the ORF was truncated was similar to the number of cases in which it

336 was not truncated, and in both cases the polymorphism patterns were consistent with neutrality

337 (Supplementary Table 5). This indicated that, for this group, the presence of a similar ORF in rat 338 does not imply functional conservation of the ORF. Therefore, we did not use information on rat 339 genomic synteny to classify the genes as conserved/non-conserved.

\section{Single nucleotide polymorphism analysis}

343 We obtained single nucleotide polymorphism (SNP) data from 20 individuals of the house 
344 mouse subspecies Mus musculus castaneus ${ }^{18}$. We classified SNPs in ORFs as non-

345 synonymous (PN, amino acid altering) and synonymous (PS, not amino-acid altering). We

346 calculated the PN/PS ratio in each ORF group by using the sum of PN and PS in all the

347 sequences ((PN/PS)obs). We calculated the expected PN/PS under neutrality ((PN/PS)exp)

348 using the mutation frequencies between pairs of nucleotides in Mus musculus castaneus and

349 the codon composition of the different sequences or sets of sequences under study

350 (Supplementary Tables 2 to 5). The observed transition to transversion ratio was 4.42, very

351 similar to the 4.26 value obtained in early observations based on mouse-rat divergence data ${ }^{57}$.

352 We tested for purifying selection by the number of observed and expected non-synonymous

353 and synonymous SNPs using a chi-square test with one degree of freedom. Positively selected

354 mutations are rapidly fixed in the population and their effect is expected to be negligible when

355 using SNP data.

\section{Proteomics data}

359 We used the proteomics database $\mathrm{PRIDE}^{21}$ to search for peptide matches in the proteins 360 encoded by various gene sets. For a protein to have proteomics evidence, we required at least

361 two distinct perfect matches of peptides that did not map to any other protein in the dataset,

362 allowing for up to two mismatches. These are very stringent conditions with a false positive rate $363<0.2 \%^{48}$.

\section{Coding score}

367 We used a previously described metric based on hexamer frequencies to calculate the coding

368 score of the sequences ${ }^{8}$. The method uses a table of pre-calculated hexamer scores that 369 measure the relative frequency of each hexamer in coding versus non-coding sequences.

370 These scores are then used to evaluate the coding propensity of a sequence based on its

371 hexamer composition. The method has been implemented in a computational program called

372 CIPHER that can be accessed online (http:// http://evolutionarygenomics.upf.edu/cipher). 


\section{Statistical tests and plots}

375

376 The generation of plots and statistical tests was performed with the $\mathrm{R}$ package ${ }^{58}$.

377

\section{Data availability}

379

380 Transcript assemblies from mouse, human and rat, as well as the mouse open reading frames

381 (ORFs) predicted to be translated have been deposited at figshare

382 (http://dx.doi.org/10.6084/m9.figshare.4702375). The code and executable file to calculate the 383 coding score can be accessed at https://github.com/jorruior/CIPHER.

384 The $\mathrm{C}$ program to calculated the PN/PS expected under neutrality is available at

385 https://figshare.com/articles/computePNPS_c/5085706. Supplementary file 1 contains

386 supplementary tables and figures.

387

\section{REFERENCES}

388 1. Kutter, C. et al. Rapid turnover of long noncoding RNAs and the evolution of gene 389 expression. PLoS Genet. 8, e1002841 (2012).

390 2. Wiberg, R. A. W. et al. Assessing Recent Selection and Functionality at Long Noncoding RNA Loci in the Mouse Genome. Genome Biol. Evol. 7, 2432-44 (2015).

392 3. Ruiz-Orera, J. et al. Origins of De Novo Genes in Human and Chimpanzee. PLOS Genet. 11, e1005721 (2015).

394 4. Ji, Z., Song, R., Regev, A. \& Struhl, K. Many IncRNAs, 5'UTRs, and pseudogenes are translated and some are likely to express functional proteins. Elife 4, e08890 (2015).

396 5. Raj, A. et al. Thousands of novel translated open reading frames in humans inferred by 397 ribosome footprint profiling. Elife 5, (2016).

398 6. Ingolia, N. T., Lareau, L. F. \& Weissman, J. S. Ribosome profiling of mouse embryonic stem cells reveals the complexity and dynamics of mammalian proteomes. Cell 147,

$400 \quad 789-802(2011)$.

401 7. Ingolia, N. T. et al. Ribosome Profiling Reveals Pervasive Translation Outside of 
403 8. Ruiz-Orera, J., Messeguer, X., Subirana, J. A. \& Alba, M. M. Long non-coding RNAs as 404 a source of new peptides. Elife 3, e03523 (2014).

405 9. Wilson, B. A. \& Masel, J. Putatively noncoding transcripts show extensive association with ribosomes. Genome Biol. Evol. 3, 1245-52 (2011).

10. Ingolia, N. T., Ghaemmaghami, S., Newman, J. R. S. \& Weissman, J. S. Genome-wide analysis in vivo of translation with nucleotide resolution using ribosome profiling. Science 324, 218-23 (2009).

11. Bazzini, A. A. et al. Identification of small ORFs in vertebrates using ribosome footprinting and evolutionary conservation. EMBO J. 33, 981-93 (2014).

412 12. Calviello, L. et al. Detecting actively translated open reading frames in ribosome profiling data. Nat Meth 13, 165-170 (2016).

414 13. Aspden, J. L. et al. Extensive translation of small ORFs revealed by Poly-Ribo-Seq. Elife e03528 (2014). doi:10.7554/eLife.03528

14. Mackowiak, S. D. et al. Extensive identification and analysis of conserved small ORFs in animals. Genome Biol. 16, 1-21 (2015).

15. Sunyaev, S., Kondrashov, F. A., Bork, P. \& Ramensky, V. Impact of selection, mutation rate and genetic drift on human genetic variation. Hum. Mol. Genet. 12, 3325-3330 (2003).

16. Gayà-Vidal, M. \& Albà, M. M. Uncovering adaptive evolution in the human lineage. BMC Genomics 15, 599 (2014). and noncoding RNA: challenges and ambiguities. PLoS Comput. Biol. 4, e1000176 (2008).

426 18. Harr, B. et al. Genomic resources for wild populations of the house mouse, Mus musculus and its close relative Mus spretus. Sci. Data 3, 160075 (2016).

428 19. Buck-Koehntop, B. A., Mascioni, A., Buffy, J. J. \& Veglia, G. Structure, dynamics, and membrane topology of stannin: a mediator of neuronal cell apoptosis induced by trimethyltin chloride. J. Mol. Biol. 354, 652-65 (2005). 
Conserved across Metazoans. PLoS Biol 14, e1002395 (2016).

433 21. Vizcaíno, J. A. et al. 2016 update of the PRIDE database and its related tools. Nucleic Acids Res. 44, D447-D456 (2016).

435 22. Slavoff, S. A. et al. Peptidomic discovery of short open reading frame-encoded peptides in human cells. Nat. Chem. Biol. 9, 59-64 (2013).

23. Toll-Riera, M. et al. Origin of primate orphan genes: a comparative genomics approach. Mol. Biol. Evol. 26, 603-12 (2009).

24. Xie, C. et al. Hominoid-specific de novo protein-coding genes originating from long noncoding RNAs. PLoS Genet. 8, e1002942 (2012).

25. Ji, Z., Song, R., Huang, H., Regev, A. \& Struhl, K. Transcriptome-scale RNase-

26. Heinen, T. J. A. J., Staubach, F., Häming, D. \& Tautz, D. Emergence of a new gene from an intergenic region. Curr. Biol. 19, 1527-31 (2009).

27. Yu, C. et al. Codon Usage Influences the Local Rate of Translation Elongation to

28. Presnyak, V. et al. Codon optimality is a major determinant of mRNA stability. Cell 160, 1111-24 (2015).

29. Tautz, D. \& Domazet-Lošo, T. The evolutionary origin of orphan genes. Nat. Rev. Genet. 12, 692-702 (2011).

30. Schlötterer, C. Genes from scratch - the evolutionary fate of de novo genes. Trends Genet. (2015). doi:10.1016/j.tig.2015.02.007 and why. Nat. Rev. Genet. 17, 567-578 (2016).

32. Okazaki, Y. et al. Analysis of the mouse transcriptome based on functional annotation of 60,770 full-length cDNAs. Nature 420, 563-73 (2002). exposes entire non-coding DNA to de novo gene emergence. Elife 5, (2016).

$$
\text { A. 112, 15690-5 (2015). }
$$

461 35. Carvunis, A.-R. et al. Proto-genes and de novo gene birth. Nature 487, 370-4 (2012). 
462 36. Zhao, L., Saelao, P., Jones, C. D. \& Begun, D. J. Origin and spread of de novo genes in 463 Drosophila melanogaster populations. Science 343, 769-72 (2014).

464 37. Wilson, B. A., Foy, S. G., Neme, R. \& Masel, J. Young Genes are Highly Disordered as Predicted by the Preadaptation Hypothesis of De Novo Gene Birth. Nat. Ecol. Evol. 1, 0146-146 (2017).

38. Plotkin, J. B. \& Kudla, G. Synonymous but not the same: the causes and consequences of codon bias. Nat. Rev. Genet. (2011). doi:10.1038/nrg2899 Nucleic Acids Res. 42, 9171-81 (2014).

40. Kaiser, C. A., Preuss, D., Grisafi, P. \& Botstein, D. Many random sequences functionally replace the secretion signal sequence of yeast invertase. Science $\mathbf{2 3 5}, 312-7$ (1987). Nature 410, 715-718 (2001).

42. Neme, R., Amador, C., Yildirim, B., McConnell, E. \& Tautz, D. Random sequences are an abundant source of bioactive RNAs or peptides. Nat. Ecol. Evol. (2017). doi:10.1038/s41559-017-0127

43. Soumillon, M. et al. Cellular source and mechanisms of high transcriptome complexity in the mammalian testis. Cell Rep. 3, 2179-90 (2013).

44. Necsulea, A. et al. The evolution of IncRNA repertoires and expression patterns in tetrapods. Nature 505, 635-40 (2014).

45. Smeds, L. \& Künstner, A. ConDeTri--a content dependent read trimmer for Illumina data. PLoS One 6, e26314 (2011).

46. Kim, D. et al. TopHat2: accurate alignment of transcriptomes in the presence of insertions, deletions and gene fusions. Genome Biol. 14, R36 (2013).

47. Pertea, M. et al. StringTie enables improved reconstruction of a transcriptome from RNA-seq reads. Nat Biotech 33, 290-295 (2015).

48. Luis Villanueva-Cañas, J. et al. New Genes and Functional Innovation in Mammals.

490 49. Gonzalez, C. et al. Ribosome profiling reveals a cell-type-specific translational landscape in brain tumors. J. Neurosci. 34, 10924-36 (2014). 
492 50. Castañeda, J. et al. Reduced pachytene piRNAs and translation underlie spermiogenic 493 arrest in Maelstrom mutant mice. EMBO J. 33, 1999-2019 (2014).

494 51. Guo, H., Ingolia, N. T., Weissman, J. S. \& Bartel, D. P. Mammalian microRNAs predominantly act to decrease target mRNA levels. Nature 466, 835-40 (2010).

496 52. Diaz-Munoz, M. D. et al. The RNA-binding protein HuR is essential for the B cell antibody response. Nat Immunol 16, 415-425 (2015).

498 53. Cho, J. et al. Multiple repressive mechanisms in the hippocampus during memory formation. Science 350, 82-87 (2015).

500 54. Sedlazeck, F. J., Rescheneder, P. \& von Haeseler, A. NextGenMap: fast and accurate read mapping in highly polymorphic genomes. Bioinformatics 29, 2790-2791 (2013).

502 55. Altschul, S. F. et al. Gapped BLAST and PSI-BLAST: a new generation of protein database search programs. Nucleic Acids Res. 25, 3389-402 (1997).

504 56. Karolchik, D. et al. The UCSC Genome Browser database: 2014 update. Nucleic Acids Res. 42, D764-D770 (2014).

506 57. Rosenberg, M. S., Subramanian, S. \& Kumar, S. Patterns of Transitional Mutation Biases Within and Among Mammalian Genomes. Mol. Biol. Evol. 20, 988-993 (2003).

508 58. R Development Core Team. R: a language and environment for statistical computing. (2016).

\section{ACKNOWLEDGEMENTS}

513 We are grateful for valuable discussions with many colleagues during this study. The work was

514 funded by grants BFU2012-36820 and BFU2015-65235-P from Ministerio de Economía e

515 Innovación (Spanish Government) and co-funded by FEDER (EC). We also received funding

516 from Agència de Gestió d'Ajuts Universitaris i de Recerca Generalitat de Catalunya (AGAUR),

517 grant number 2014SGR1121.

\section{FIGURE LEGENDS}

521 Figure 1. Detection of translated ORFs. a. Workflow to identify translated ORFs. Ribosome profiling 
522 (Ribo-Seq) reads, corresponding to ribosome-protected fragments, are mapped to all predicted canonical

523 ORFs with length $\geq 30$ nucleotides in transcripts. This is performed with single-nucleotide resolution after

524 computing the read P-site per each read length. In each ORF, reads per frame and read uniformity are

525 evaluated by RiboORF. b. Number of translated and non-translated expressed genes belonging to

526 different classes after integrating data from eight different mouse tissues (Supplementary Table 1). c.

527 Number of translated ORFs belonging to different classes. The translated ORFs have been divided into

528 small ORFs (smORF, < 100 aa) and long ORFs ( $\geq 100 \mathrm{aa}$ ), depending on their length.

530 Figure 2. Identification of selection signatures. a. Workflow to identify conserved and non-conserved

531 ORFs. Translated ORFs shorter than 24 amino acids, as well as non-conserved upstream and

532 downstream ORF in conserved transcripts (uORFs and dORFs, see Methods), were filtered out. Any ORF

533 with at least one BLAST match in another species was classified as conserved (C), otherwise it was

534 classified as non-conserved (NC). b. Coding score in conserved (C) and non-conserved ORFs (NC).

535 Conserved ORFs showed significantly higher coding score values than non-conserved ones; ${ }^{* * *}$ Wilcoxon

536 test, $p$-value $<10^{-5}$. Non-conserved ORFs with a high coding score value $(\geq 0.1014)$ were classified as

$537 \mathrm{NC}-\mathrm{H}$, and the rest were classified as NC-L. c. Analysis of selective constraints in translated ORFs. PN/PS

538 (obs/exp) refers to the normalized ratio between non-synonymous (PN) and synonymous (PS) single

539 nucleotide polymorphisms; a value of 1 is expected in the absence of selection at the protein level.

540 Conserved and NC-H ORFs showed significant purifying selection signatures. In contrast, NC-L ORFs did

541 not show evidence of purifying selection at the protein level. Many conserved ORFs in IncRNAs are likely

542 to encode functional micropeptides. Differences between observed and expected PN/PS were assessed

543 with a chi-square test, ${ }^{*} \mathrm{p}$ - value $<0.05,{ }^{* * *} \mathrm{p}$-value $<10^{-5}$. Error bars indicate the standard error of the

544 sample proportion. Numbers of ORFs for the different categories are also displayed. d. Distribution of

545 normalized PN/PS values for individual ORFs in different gene classes. Only ORFs with at least 10 SNPs

546 were considered; the NC-H group contained too few cases to be analysed. The differences between $\mathrm{C}$ and

547 NC-L are significant (Wilcoxon test, $p$-value $<10^{-5}$ ).

549 Figure 3. Three nucleotide periodicity of translated ORFs. The mapping of Ribo-Seq reads on different

550 types of ORFs is shown. The $Y$ axis represents the log-number of reads, the $X$ axis the positions in the

551 ORF. The reads show strong frame bias in the functional (conserved) and the neutral (NC-L) examples,

552 with a preponderance of in-frame reads (green) versus off-frame reads (red and blue), while the frame bias

553 is randomly distributed in the negative control (SNORA18). The exon/intron structure and the amino acid

554 sequence for translated ORFs is also shown. 
556 Figure 4. Properties of neutrally evolving ORFs. a. Relationship between the percentage of reads

557 falling in the correct frame in neural embryonic stem cells cells and hippocampus samples, for neutral and

558 functional ORFs having at least 10 reads in both samples and being translated in at least one sample.

559 Spearman correlation coefficient is $R=0.4224$ for the neutrat set ( $p$-value $<10^{-5}$ ) and $R=0.4360$ for the

560 functional set $\left(p-v a l u e<10^{-5}\right)$. b. Distribution of read periodicity, read uniformity and RibORF scores in

561 neutral and functional translated ORFs after controlling for the number of Ribo-Seq reads and size of

562 ORFs. The 'functional normalized' set is a randomly taken subset of the functional ORFs that has the

563 same number of mapped Ribo-Seq reads and ORF size distribution as the set of neutrally evolving ORFs

$564(n=900)$. Data is represented as box-plots for different number of read intervals; the box contains $50 \%$ of

565 the data, horizontal line is the median value.

566

567 Figure 5. Factors influencing the translation of neutrally evolving ORFs. a. Influence of coding score

568 in the translatability of neutrally evolving ORFs. Translated ORFs showed significantly higher coding score

569 than non-translated ORFs, both sets had significantly higher coding scores than introns (Wilcoxon test p-

570 value $<10^{-5}$, indicated by ***). b. Influence of coding score in the translatability of ORFs controlling for

571 gene expression values, the two sets have comparable maximum FPKM gene expression (median FPKM

572 value $=11.10$ ). Translated ORFs showed significantly higher coding score values than non-translated

573 ORFs; (Wilcoxon test $p$-value $<10^{-5}$ ). c. Influence of maximum FPKM gene expression and ORF length in

574 the translatability of neutral ORFs normalized by coding score (median coding score value $=-0.0052$ ).

575 Translated ORFs showed significantly higher FPKM values than non-translated ORFs (Wilcoxon test p-

576 value $<10^{-5}$ ); differences in length were not significant. 
bioRxiv preprint doi: https://doi.org/10.1101/064915; this version posted February 22, 2018 . The copyright holder for this preprint (which was not certified by peer review) is the author/funder, who has granted bioRxiv a license to display the preprint in perpetuity. It is made available under aCC-BY-NC-ND 4.0 International license.

\section{FIGURES}

\section{$579 \quad$ Figure 1}

580

a

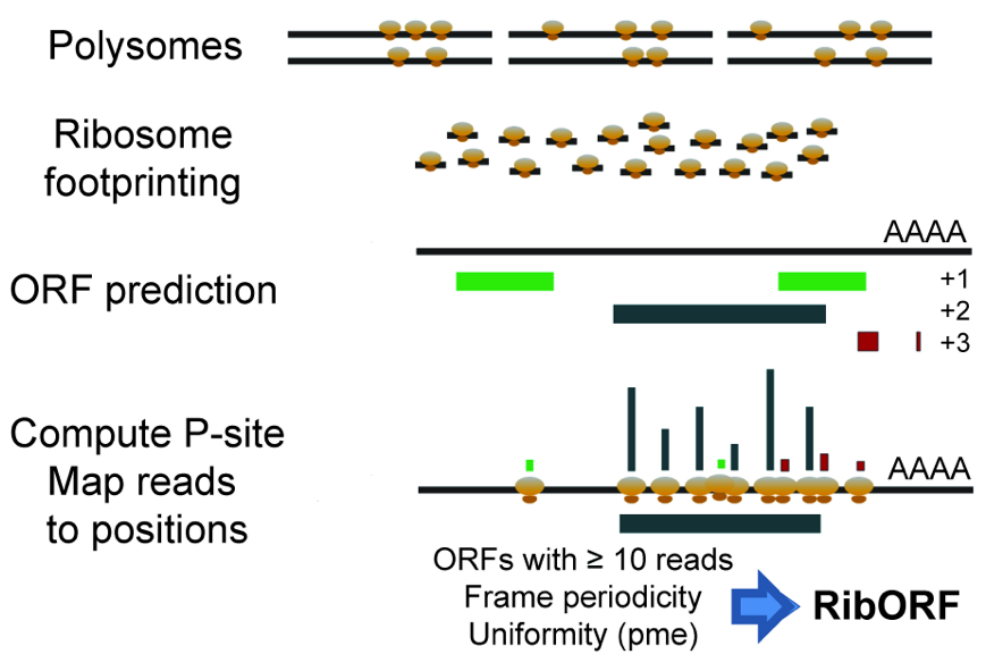

b

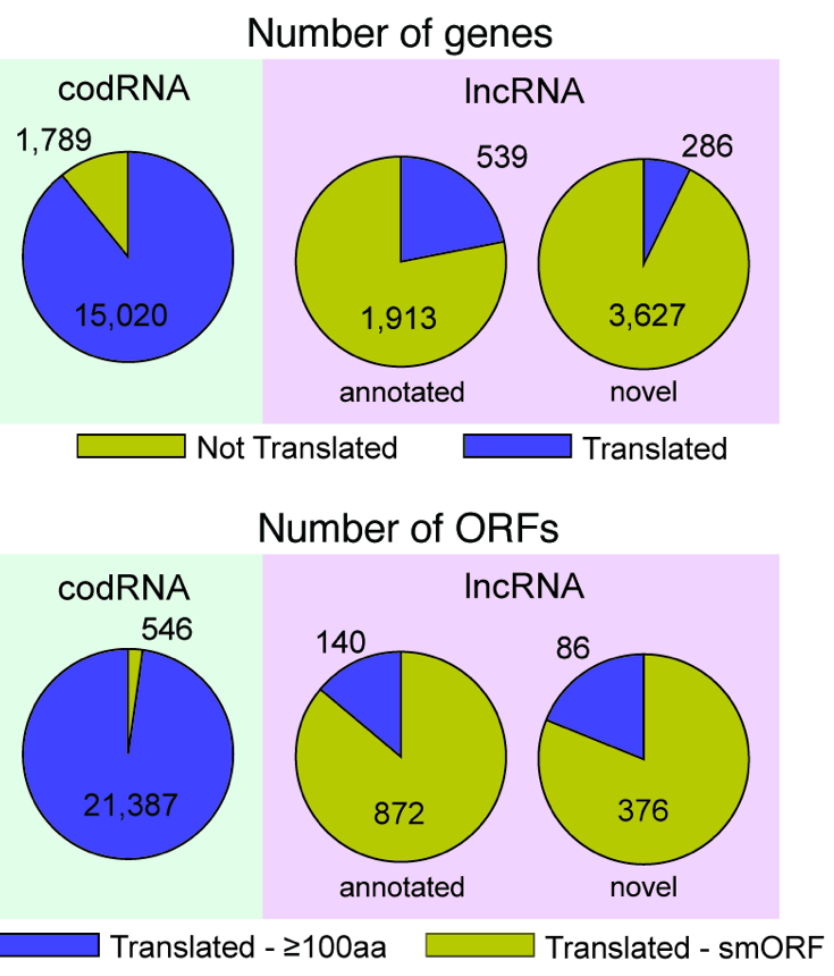


bioRxiv preprint doi: https://doi.org/10.1101/064915; this version posted February 22, 2018 . The copyright holder for this preprint (which was not certified by peer review) is the author/funder, who has granted bioRxiv a license to display the preprint in perpetuity. It is made available under aCC-BY-NC-ND 4.0 International license.

\section{Figure 2}

584

a

Translated ORFs

Filter out uORFs, dORFs and ORFs $<24$ amino acids (4,384 cases)

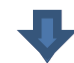

BLAST against:

a) human and rat transcriptomes

b) annotated proteomes for 101 species

Conserved (C) Non-conserved (NC) b

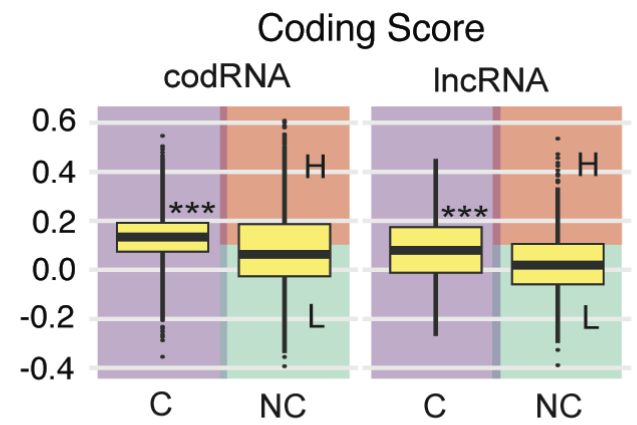

NC-H: Non-conserved - High score (coding score $\geq 0.1014$ )

NC-L: Non-conserved - Low score (coding score < 0.1014)
C
Purifying selection in ORFs

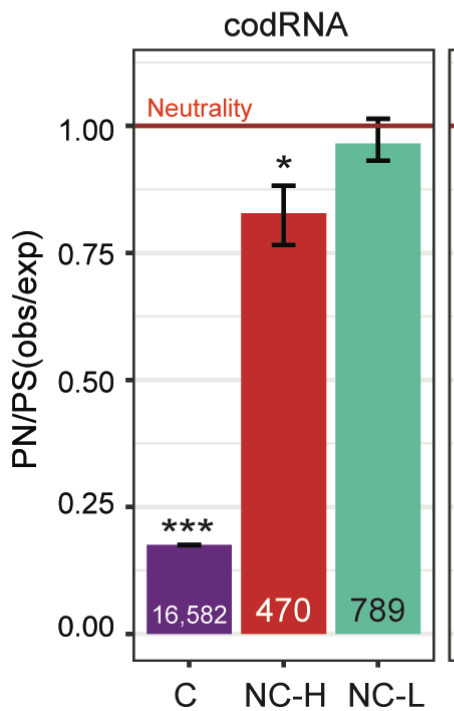

IncRNA

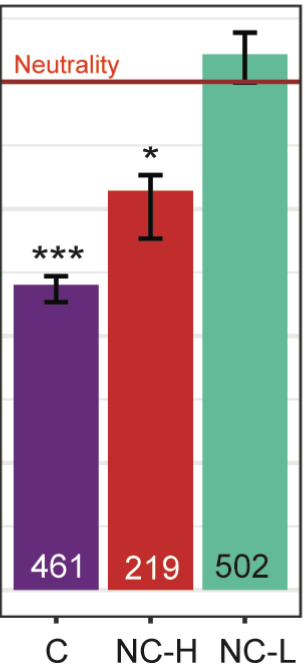

d

Purifying selection ORFs $\geq 10$ SNPs

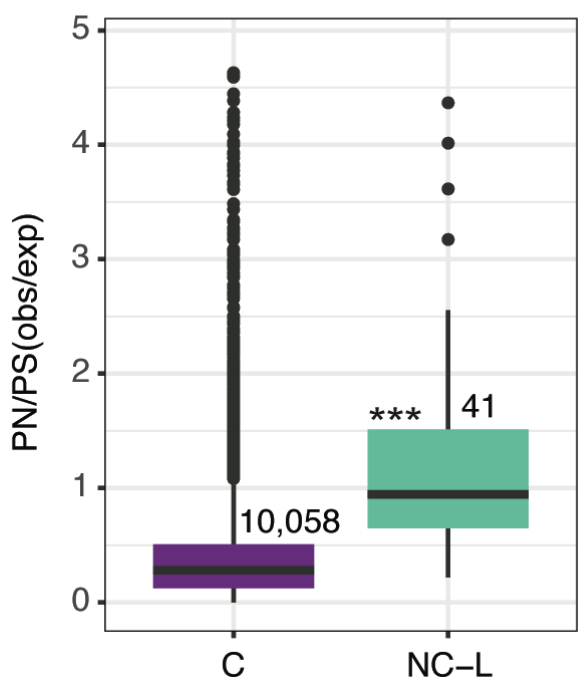


bioRxiv preprint doi: https://doi.org/10.1101/064915; this version posted February 22,2018 . The copyright holder for this preprint (which was not certified by peer review) is the author/funder, who has granted bioRxiv a license to display the preprint in perpetuity. It is made available under aCC-BY-NC-ND 4.0 International license.

Stannin (Functional)

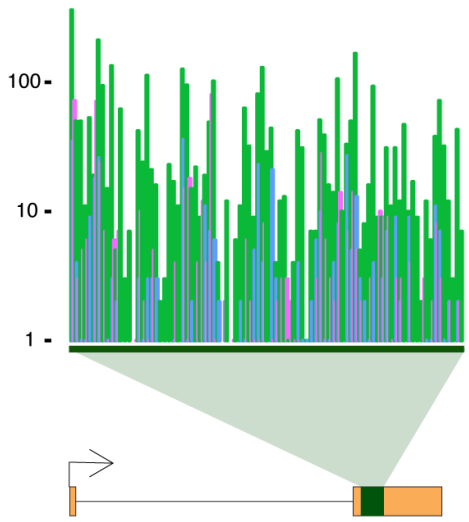

MS IMDHS PTTGVVTVIVILIAIAALGALIL GCWCYLRLORISOSEDEESIVGDGETKEPF LLVQYSAKGPCVERKAKLMTANSPEVHG
XLOC_007614 (Neutral)

$1-$

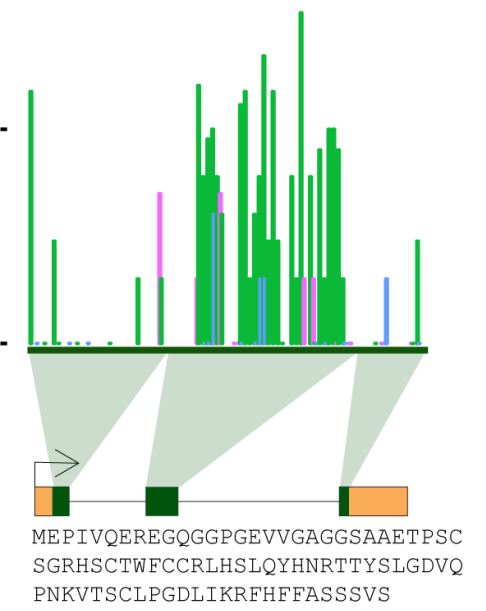

SNORA18 (snoRNA)

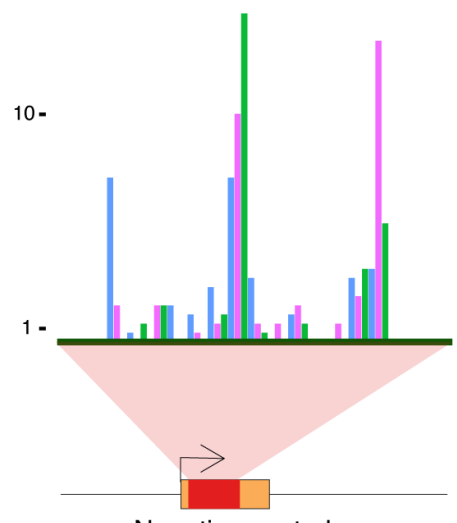

Negative control 
bioRxiv preprint doi: https://doi.org/10.1101/064915; this version posted February 22, 2018. The copyright holder for this preprint (which was not certified by peer review) is the author/funder, who has granted bioRxiv a license to display the preprint in perpetuity. It is made available under aCC-BY-NC-ND 4.0 International license.

\section{$591 \quad$ Figure 4}

a

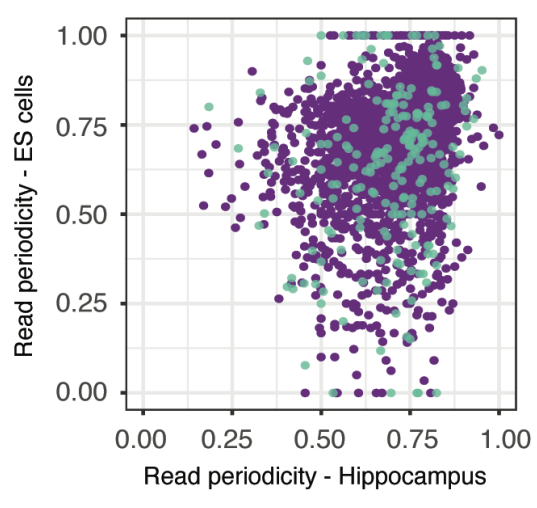

592

b

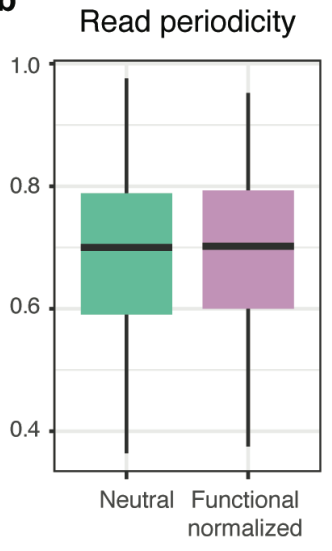

Read uniformity

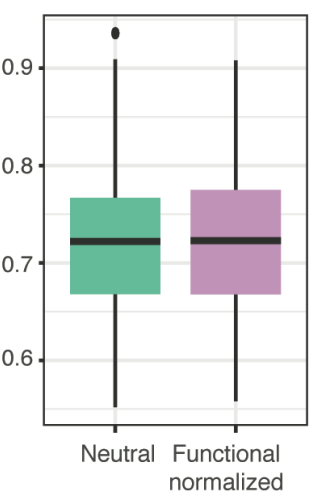

RibORF score

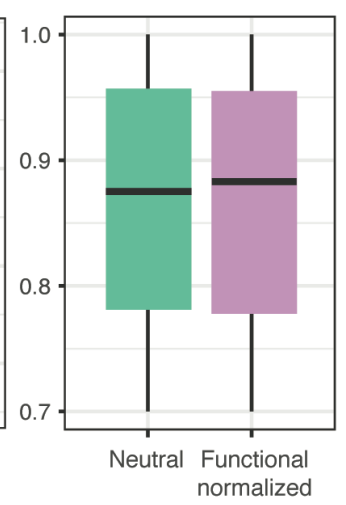

593 
bioRxiv preprint doi: https://doi.org/10.1101/064915; this version posted February 22, 2018 . The copyright holder for this preprint (which was not certified by peer review) is the author/funder, who has granted bioRxiv a license to display the preprint in perpetuity. It is made available under aCC-BY-NC-ND 4.0 International license.

\section{$594 \quad$ Figure 5}

595

a

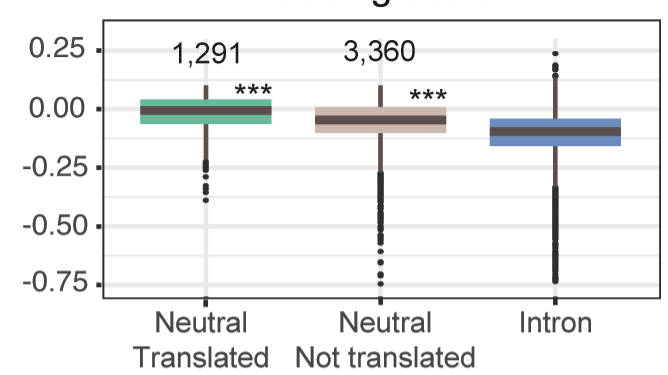

b

Normalized by FPKM

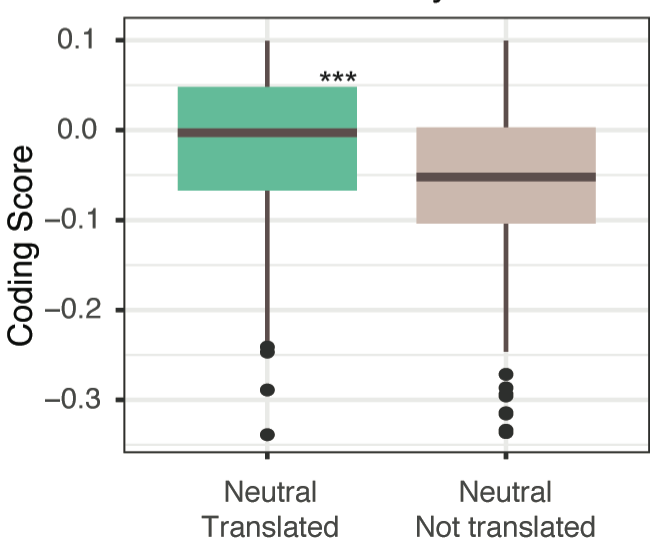

c Normalized by coding score
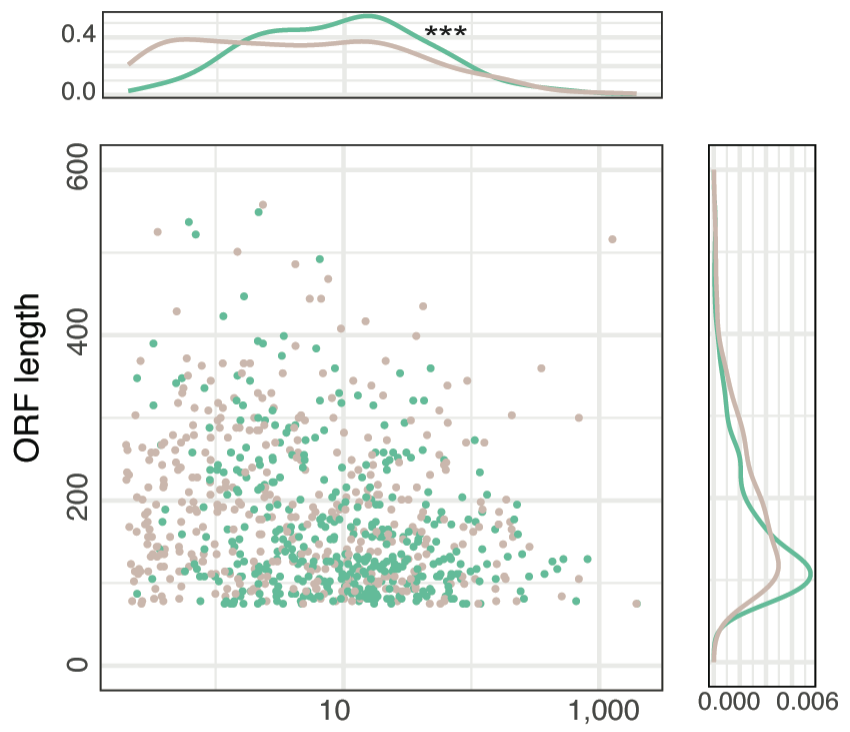

$\log$ FPKM

Neutral - Translated

Neutral - Not translated 